\title{
Malaria-Prophylaxe und kolonialer Städtebau: Fortschritt als Rückschritt?
}

\author{
Von Albert Wirz
}

Die Malaria ist nicht nur eine der am weitesten verbreiteten, sondern auch eine der ältesten und gefährlichsten Infektionskrankheiten. Wenn sie noch zu Anfang des letzten Jahrhunderts auch in großen Teilen Europas endemisch war - selbst einzelne Gebiete der Schweiz waren davon betroffen -, so beschränkt sich ihre Ausbreitung heute im wesentlichen auf die Tropengebiete. Dort freilich fordert sie nach wie vor einen gewaltigen Blutzoll. Nach Angaben der Weltgesundheitsorganisation lebt etwa die Hälfte der Weltbevölkerung in malariagefährdeten Gebieten. Die Zahl der jährlichen Erkrankungen wurde 1974 auf 120 Millionen geschätzt, die der Todesfälle auf ein bis zwei Millionen. Betroffen sind nicht zuletzt Kinder. Sorgfältigen Schätzungen zufolge ist die Malaria in Afrika, wo sie in ihrer schwersten Form der Malaria tropica auftritt, für zehn und mehr Prozent der Todesfälle bei Kindern und Jugendlichen verantwortlich ${ }^{1}$. Und wenn im letzten Jahrhundert Westafrika als «Grab des weißen Mannes» galt, wie der Titel eines journalistischen Berichts über die englische Kronkolonie Sierra Leone aus dem Jahre 1836 lautet, dann war das ebenfalls zu einem guten Teil eine Folge der Malaria und der Unkenntnis ihrer genauen Verursachung ${ }^{2}$.

Man kann sich fragen, ob die koloniale Unterwerfung Afrikas überhaupt möglich gewesen wäre, wenn nicht zu Ende des letzten Jahrhunderts bahnbrechende wissenschaftliche Entdeckungen nach Jahrhunderten blinden Tappens der Ärzte auf einmal Klarheit geschaffen, die wahre Pathogenese aufgeklärt und damit eine rationale und wirksame Malaria-Bekämpfung ermöglicht hätten. Umgekehrt aber wird man sich fragen, ob diese Entdeckungen wirklich dem Fortschritt eine Bahn gebrochen oder ob nicht die soziopolitischen und kulturellen Rahmenbedingungen den wissenschaftlichen Fortschritt in sozialen Rückschritt umgebogen haben. In diesem Sinne soll im folgenden zuerst der Weg aufgezeigt werden, der zurückgelegt werden mußte, um die Ursachen der Malaria zu erkennen, um dann in einem zweiten Schritt die aus dem jeweiligen Wissen abgeleiteten ProphylaxeMethoden einander gegenüberzustellen und sie einzuordnen in die allge- 
meine Geistesgeschichte. Abschließend soll am Beispiel der Stadt Duala (Kamerun) dargelegt werden, wie im kolonialen Städtebau die wissenschaftlichen Denkmodelle in eine räumliche Ordnung übergeführt wurden.

Obwohl seit Jahrhunderten beobachtet, beschrieben und bekämpft, gab die Malaria noch 1880 Anlaß zu wildesten Spekulationen ${ }^{3}$. Zwar hatte bereits der römische Schriftsteller Marcus Terentius Varro (116-27 v. Chr.), um nur einen antiken Autor zu nennen, in seinem Alterswerk Rerum rusticarum kleine, unsichtbare, in Sümpfen lebende Tiere als mögliche Träger der Krankheitskeime genannt. Noch näher an der Wahrheit waren die Shambaa der Gegend von Usambara im heutigen Tansania. Sie bezeichneten mit dem Worte $m b u$ sowohl Mücken wie auch Fieberkrankheiten, die sie sich regelmäBig bei ihren Handelsreisen vom Hochland an die Küsten holten und die die Europäer dann als Malaria diagnostizierten ${ }^{4}$. Medizinhistoriker verweisen zudem auf die schon von Herodot beobachtete Gewohnheit ägyptischer Fischer, sich am Ufer des Nachts durch ihre Netze vor Mückenstichen zu schützen. Ebenso könnte man auf afrikanische Viehhirten verweisen, die ihr Nachtlager nahe bei ihren Tieren aufschlugen, weil sie wußten, daß die Mücken, vom Vieh angezogen, sie weniger belästigen würden. Daraus läßt sich allerdings nicht ablesen, da $\beta$ die Betroffenen oder allgemein die Volksmedizin einen Kausalzusammenhang zwischen Mücken und Fieberkrankheiten gesehen hätte.

Denn auch in einem Vademecum für Europäer in Indien aus dem Jahre 1878 wird den Lesern geraten, sich tagsüber durch die ortsüblichen großen Fächer (punkah) und nachts durch Bettvorhänge vor Moskitos zu schüt$z^{2}{ }^{5}$. Doch diese Maßnahmen wurden keineswegs im Sinne einer MalariaProphylaxe empfohlen, sondern bloß als Schutz vor einer zwar äußerst lästigen, sonst aber harmlosen Plage. Hinsichtlich der Verursachung der Malaria erweisen sich die Verfasser als Anhänger der damals allgemeingültigen Sumpf- oder Miasma-Theorie, die auch im Namen zum Ausdruck kommt, den die deutschen und französischen Zeitgenossen dem durch Schüttelfröste, intermittierende Fieber, Anämie und druckempfindliche Milz gekennzeichneten Krankheitsbild gaben: Sumpffieber bzw. paludisme. Der vom italienischen mala aria oder schlechte Luft hergeleitete Begriff Malaria bezeichnete weniger die Krankheit als die vermutete Ursache der Fieber. 
So nennt etwa James Africanus Horton in seinem 1874 in London veröffentlichen Buch The diseases of tropical climates and their treatment als eigentlichen Verursacher die «Ausdünstung eines gewissen unsichtbaren Effluviums von zerfallener vegetabilischer oder tierischer Materie ..., die man ... als Malaria bezeichnet». ${ }^{6}$ Während Horton einschränkend darauf hinweist, daß dieses Effluvium oder Gift nur in seiner Auswirkung auf die körperliche Verfassung der Patienten erkannt werden könne, wußte der Verfasser des bereits genannten Vademecums, daß das Malaria-Gift vor allem zu Beginn der Regenzeit auftrete, am stärksten jeweils zwischen Sonnenuntergang und Sonnenaufgang wirke, überdies daß es schwerer als Luft sei und sich mit Bodennebeln vermenge, weshalb es seiner Ansicht nach nichts Gefährlicheres gab, als im Freien auf dem Boden zu schlafen. Hingegen empfahl er das Anpflanzen hoher weitausladender Bäume, da sich in deren Laubwerk das Gift dann verfange ${ }^{7}$. Als wichtigste Quelle des vermuteten Gifts betrachteten Horton wie Mair, und mit ihnen praktisch die gesamte europäische Ärzteschaft, Sümpfe, stehende Wasser und «faule Uferstriche». Mücken kamen in ihren Überlegungen nicht vor.

Alle diese Theorien sind, wie wir heute wissen, falsch. Sie gründeten auf richtigen Beobachtungen wie denen, daß Malaria im jahreszeitlichen Wechsel unterschiedlich stark auftritt und daß vor allem Sumpfgebiete und tropische Regionen mit hoher Luftfeuchtigkeit malariaverseucht waren. Die Schlußfolgerungen allerdings waren falsch und ohne empirische Grundlage. Der Umstand, daß auch Schiffsbesatzungen auf hoher See oder die Bewohner einzelner Gebirgsgegenden an Malaria erkranken konnten, nährte zwar Zweifel an der Allgemeingültigkeit der Theorie, vermochte sie jedoch keineswegs aus den Angeln zu heben. Zum Dogma verhärtet, verdeckte sie mehr, als daß sie erklärte. Der Durchbruch zu einem neuen Paradigma gelang erst, als die im Verlaufe des 19. Jahrhunderts entwickelte Bakteriologie völlig neue Frageansätze ermöglichte.

Entscheidend war hier zum einen die Entdeckung von Protozoen im Blute Malariakranker. Dieser Nachweis gelang dem französischen Militärarzt Alphonse Laveran 1880 in Algerien. Noch war aber die Frage der Übertragung ungeklärt. Diese Lücke vermochte achtzehn Jahre später der englische Militärarzt Ronald Ross zu schließen, der in Indien arbeitete. Ross ging bei seinen Untersuchungen vom Verhalten verwandter Blutparasiten der Vögel aus und entdeckte, daß dieselben einen doppelten Entwicklungskreislauf haben, von dem sich der eine, ungeschlechtliche, im Blutkreislauf des Vogels, der andere, geschlechtliche, aber im Körper weiblicher Stech- 
mücken der Gattung Anopheles vollzieht. Der Gedanke an eine Analogie zur Entwicklung der Malaria-Plasmodien lag nahe und konnte denn auch bald danach vom italienischen Zoologen Giovanni Grassi empirisch belegt werden. Grassi gelang zudem die Isolierung der für die einzelnen MalariaFormen verantwortlichen Plasmodienarten. Damit war die spekulative Sumpftheorie, die für Jahrhunderte Gültigkeit gehabt hatte, ein für allemal widerlegt und durch wissenschaftliche, überprüfbare Erkenntnisse ersetzt. Ronald Ross und Alphonse Laveran wurden 1902 bzw. 1907 mit dem Nobelpreis geehrt; der Engländer wurde zudem 1911 für seine Verdienste geadelt $^{8}$.

Trotzdem möchte ich nochmals auf die Sumpftheorie zurückkommen. Ihre Hypothesen und Schlußfolgerungen waren zwar nachweislich falsch; doch die aus ihnen abgeleiteten Methoden für den Umgang mit der Malaria waren - abgesehen vielleicht von der verheerenden Quecksilber-Therapie weder unsinnig noch ein Hemmnis für den Fortschritt, wenn wir darunter das Wohlergehen einer möglichst breiten Schicht von Leuten und die Errichtung einer offenen Gesellschaft verstehen, in der Können und Wissen für den Statuserwerb wichtiger sind als Geld und Herkunft.

\section{III}

Es ist nicht zu leugnen, daß die beschränkten Kenntnisse hinsichtlich der Malaria-Ursachen die Ärzte zu einem gewissen Fatalismus zwangen. Die meisten Ärzte konzentrierten ihre Ratschläge darauf, die Leute zu einer gesunden Lebensführung anzuhalten, wobei Mäßigung in allen Dingen das Richtmaß gab. Darüber hinaus erteilten sie den Rat, sich höchstens zeitweise an ungesunden Orten niederzulassen und immer wieder zwischendurch gesunde Orte aufzusuchen, wobei sie vor allem an Höhenkurorte dachten. Weiterreichend waren Vorschläge, die Umwelt selbst zu verändern, sei es durch die Trockenlegung von Sümpfen oder die Sanierung der Kanalisations- und Abwasserverhältnisse in den betroffenen Städten. Doch merkwürdigerweise spielten hier die Ärzte nur eine untergeordnete Rolle. In diesen Belangen waren sie weniger Anreger als Nutznießer und Beobachter, die feststellten, wie die aus wirtschaftlichen Überlegungen heraus vorangetriebene Trockenlegung von Sümpfen und die Verbesserung der hygienischen Verhältnisse in den Städten im Verlaufe des 19. Jahrhunderts einen merklichen Rückgang der Malaria in weiten Teilen Europas zur Folge 
hatten. In Afrika und in anderen Tropengebieten war die Hilflosigkeit der medizinsichen Zunft besonders groß. Doch gerade diese Hilflosigkeit, so scheint es, hat fortschrittliche Gedanken ermöglicht.

Bereits 1768 nämlich hat der leitende Arzt des königlichen MarineLazaretts von Haslar bei Portsmouth, James Lind, eine der großen tropenmedizinischen Autoriäten seiner Zeit, den Vorschlag gemacht, für den Handel an ungesunden Orten der Guinea-Küste «einige junge Eingeborene» auszubilden. Deren Loyalität wollte er durch «gehörige Entlohnung», besondere Vorrechte, Ehrenstellen und schließlich die Aufnahme ins englische Bürgerrecht sichern: «Es sollte ihnen zugestanden werden, zu einem gewissen Range in der Regierung oder Armee zu steigen; und sie sollen alle die Vorrechte wie die englischen Untertanen genießen.» Als Vorbild galten ihm die portugiesischen Kolonien, wo die «Schwarzen und Halbmohren ... sich alle als so treue und gute Untertanen der Krone Portugals als irgendwelche in Lissabon beweisen». ${ }^{9}$ Es ist zu beachten, daß diese Vorschläge zu einer Zeit gemacht wurden, als Afrika für die Europäer nicht mehr war als ein «Gehege für die Handelsjagd auf Schwarzhäute», wie Karl Marx es nannte. Das heißt: die Europäer verschacherten Afrikaner als Sklaven nach Amerika, und doch waren sie nicht notwendigerweise Rassisten; der unmenschliche Menschenhandel schloß Gedanken nicht aus, die von einem Gleichheitsideal aller Menschen getragen waren.

Die von Lind angestellten Überlegungen blieben vorerst zwar ohne Folgen. Als aber England in der ersten Hälfte des 19. Jahrhunderts im Zeichen des Kampfes gegen den Sklavenhandel seine Präsenz in Westafrika verstärkte - durch die Stationierung von Marine- und Armee-Einheiten einerseits und die Errichtung erster Kolonien andererseits -, da wurde auch die anhaltend hohe Sterblichkeit unter den Europäern zu einem vordringlichen Problem, das nicht zuletzt die Politiker beschäftigte. Und um den Bedürfnissen der westafrikanischen Garnisonen nach ärztlicher Versorgung besser gerecht zu werden, verfiel das englische Kriegsministerium 1854 auf eine Lösung, wie sie in Linds Gedankengängen vorgezeichnet war: es bat die seit einem halben Jahrhundert in Sierra Leone tätige Church Missionary Society, geeignete Afrikaner für den Dienst im Army Medical Corps auszubilden. So kam es, daß 1855 drei Afrikaner zu Medizinstudien nach England geschickt wurden. Einer davon war der zwanzigjährige James Africanus Horton. Horton war der Sohn eines von der englischen Flotte befreiten und danach in Sierra Leone angesiedelten Igbo-Sklaven. Nach vierjährigen Hochschulstudien promovierte James Africanus Horton 1859 in Edinburg 
zum Dr.med. Hierauf diente er während zwanzig Jahren als Armeearzt an der Goldküste und in Gambia ${ }^{10}$.

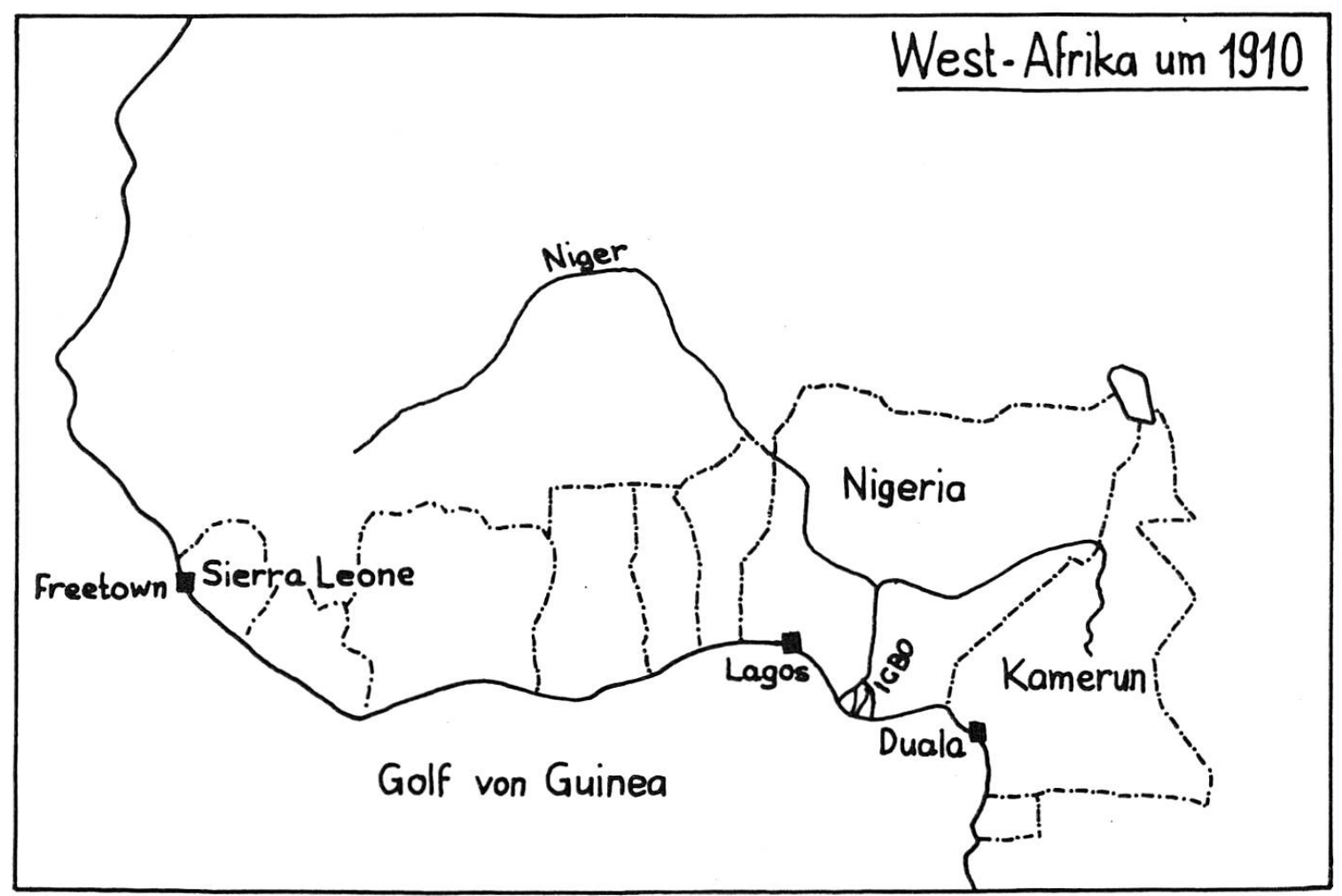

Als er 1880 um seinen Abschied bat, bekleidete er den Rang eines Oberstleutnants. Er hatte nicht nur in den Ashanti-Kriegen von 1863/64 und 1873/74 gekämpft, sondern zeitweise auch als Kommandant von Sekondi gewirkt, was ihm sogar Befehlsgewalt über weiße Engländer verliehen hatte. Darüber hinaus war Horton ein äußerst produktiver Publizist und Wissenschaftler. Er verfaßte nicht weniger als acht Bücher, die außer medizinischen, botanischen und geologischen Problemen namentlich politischen und sozialen Fragen der westafrikanischen Völker gewidmet waren ${ }^{11}$. Unter anderem entwarf er den Plan einer schrittweisen Entwicklung der britischen Westafrika-Kolonien zur Selbstregierung nach englischem Muster. Noch ehe die Kolonisation Afrikas richtig begonnen hatte, dachte er bereits an die Dekolonisation. Der Plan sollte Mitte dieses Jahrhunderts Wirklichkeit werden. So lange sollte es auch dauern, bis Afrikaner wieder in ähnlich hohe Positionen aufsteigen konnten wie Horton. Für unseren Zusammenhang wichtig ist aber vor allem der in Hortons Büchern aufscheinende Zusammenhang zwischen Bildung und Freiheitsverlangen. 
Hortons Karriere ist ganz außergewöhnlich, vor allem wenn man bedenkt, daß den europäischen Zeitgenossen die Igbo zusammen mit den Angehörigen anderer segmentär organisierter Gesellschaften in Afrika als Inbegriff wildesten Barbarentums, als Primitivste der Primitiven galten. Seine Laufbahn ist aber keineswegs einmalig. Vielmehr ist sie beispielhaft für die Möglichkeiten am Vorabend der kolonialen Unterwerfung, als die Interessen der Philanthropen und der Kaufleute sich für einmal ergänzten. Damit ist angedeutet, daß bis zur Jahrhundertwende die Voraussetzungen für schulischen und beruflichen Aufstieg der Afrikaner sich von Grund auf änderten, und zwar keineswegs zum Vorteil der Afrikaner. In der Tat hat sich bereits Horton einer wachsenden Grundwelle von Rassismus gegenübergesehen. In diesem Zusammenhang ist an das Erscheinen von zwei Büchern zu erinnern, die dann während Jahrzehnten meinungsbildend bleiben sollten: an das 1850 veröffentlichte Buch Races of men des englischen Mediziners Robert Knox und den drei Jahre später in Paris verlegten Essai sur l'inégalité des races humaines des französischen Diplomaten Arthur Comte de Gobineau.

Beide Autoren glaubten, in der Rasse den Schlüssel zum Verständnis alles menschlichen Tuns gefunden zu haben, wobei sie die Ansicht vertraten, die verschiedenen Rassen stünden in einem unveränderbaren, hierarchischen Verhältnis zueinander, mit den Europäern bzw. den Angelsachsen an der Spitze. Horton seinerseits bezweifelte nicht, daß die Europäer des 19. Jahrhunderts den Gipfel der Zivilisation erreicht hätten, während die Afrikaner noch immer mehrheitlich in einer Nacht der Barbarei verharrten. Als Folge seiner Ausbildung hatte er das europäische Zivilisationsideal und Rassendenken völlig in sich aufgenommen. Doch im Unterschied zu Knox und Gobineau wollte er den Rassencharakter nicht als eine feste, unverrückbare Kategorie verstanden wissen, sondern als eine durch Erziehung und Umwelt bestimmte und folglich veränderbare Größe. Für Hortons Denken zentral war die Überlegung, daß alle Rassen und Völker, nicht zuletzt auch die Afrikaner, durch Schulung zur Zivilisation gebracht werden könnten ${ }^{12}$. Wer möchte in Anbetracht seiner Leistungen und derjeniger anderer Afrikaner bezweifeln, daß diese Meinung im Gegensatz zu jener der Anhänger Gobineaus oder Knox' auf empirischen Grundlagen beruhte. Trotzdem setzten sich deren Ideen durch, während Hortons Leistungen zunehmend in Vergessenheit gerieten. 
Die Gründe brauchen hier nicht im einzelnen erörtert zu werden. Immerhin kann nochmals darauf verwiesen werden, daß Bildung und Unfreiheit sich auf die Länge ausschließen. Daß Bildung das Verlangen nach politischer Partizipation weckt, läßt sich nicht nur an der Biographie von James Africanus Horton ablesen; es ist dies auch ein Problem, dessen sich die Kolonialherren sehr wohl bewußt waren. Ihr Wille zur Ermöglichung sozialen Wandels in Afrika war deshalb in engen Grenzen gefangen, obwohl sie selbst ihre Präsenz in Afrika als Zivilisationsmission zu rechtfertigen suchten. Der Ausblick in die allgemeine Geistesgeschichte des 19. Jahrhunderts ist für eine Analyse der Malaria-Prophylaxe insofern von entscheidender Bedeutung, als jede Prophylaxe nicht nur den Willen zu Veränderungen auf seiten der Fachleute voraussetzt, sondern auch den Glauben an die Lernfähigkeit der betroffenen Menschen. Die Tragik der Geschichte will es, daß zu dem Zeitpunkt, als eine wissenschaftliche Revolution erstmals eine rationale Malaria-Bekämpfung und -Prophylaxe möglich machte, im gesellschaftlichen Bereich rassistisches Herrendenken an die Stelle des früheren aufklärerischen Denkens getreten war. Profitiert haben davon letztlich nur die Mücken.

Der von Ross entdeckte Moskito-Zyklus eröffnete im wesentlichen vier Möglichkeiten im Kampf gegen die Malaria. Man konnte (1.) die Brutstätten der Anopheles-Stechmücken zerstören oder (2.) gegen die Anopheles selbst vorgehen und sie auszurotten versuchen, wenn man sich nicht darauf beschränken wollte, (3.) die Menschen vor Mückenstichen zu schützen. Schließlich gab es (4.) die Möglichkeit, die Plasmodien-Entwicklung im menschlichen Körper selbst zu unterbrechen. Keine dieser Strategien war wirklich neu. Die Zerstörung der Brutstätten entsprach den Assanierungsprogrammen im Europa des 19. Jahrhunderts. Das Vorgehen gegen die Mücken selbst war nichts anderes als die Systematisierung dessen, was jeder einzelne tut, wenn er einer Mückenplage ausgesetzt ist. Der vierte Weg entsprach der vorbeugenden Einnahme von Chinin, das seit dem 17. Jahrhundert zumindest im spanischen Kulturbereich wegen seiner heilenden Wirkung bei Sumpf- und Wechselfiebern geschätzt wurde. Robert Koch, einer der um die Entwicklung der Bakteriologie besonders verdienten Pioniere, ist es, der in systematischen Versuchen auf Neu-Guinea die genaue 
Wirkungsweise des aus der Rinde des Cinchonia-Baums gewonnenen Chinins wissenschaftlich untersucht und erhärtet hat.

Den verschiedenen Strategien gemeinsam war das Problem, daß sie in ihrer Durchführung kostspielig waren, nicht ohne aktive Beteiligung der breiten Bevölkerung auskamen und trotz des anfänglichen Optimismus einzelner Forscher nur langfristig und im Verein mit tiefgreifenden Strukturreformen Erfolg versprachen. Denn, wie man in Italien um die Jahrhundertwende erkannte, die Malaria ist mehr als ein medizinisches bzw. biologisches Problem: sie ist eine soziale Krankheit. Der große italienische Malariaforscher Angelo Celli sprach von einem Teufelskreis von Malaria und Armut, den es zu durchbrechen gelte ${ }^{13}$. Einer Übertragung dieser Erkenntnis auf Afrika, wo die Malaria besonders virulent war, stand nicht nur der Rassismus der Kolonialherren entgegen, sondern auch die kurzsichtigen Profitinteressen der Europäer, die alle unproduktiven Investitionen scheuten und darauf drängten, daß die Kolonien sich selbst finanzierten. Für soziale Verbesserungen gab es in den Kolonien noch weniger Geld als in Europa.

Um so verdienstvoller erscheint die Politik, die der Gouverneur von Lagos, der Arzt William MacGregor, um die Jahrhundertwende verfolgte. Trotz beschränkter Mittel leitete er in Lagos ein langfristiges Sanierungsprogramm in die Wege, das außer der Trockenlegung von Sümpfen den Aufbau eines städtischen Gesundheitswesens vorsah und die fehlenden Mittel durch die Mobilisierung der einheimischen Bevölkerung wettzumachen versuchte. Besonders stolz war er auf die karitativen Aktivitäten der Lagos Ladies' League, eines losen Zusammenschlusses von Damen aus der afrikanischen Oberschicht. MacGregor war sich bewußt, daß Fortschritte auf diese Weise nur langsam greifbar sein würden, doch er glaubte, daß die so erreichten Erfolge dann um so sicherer seien und daß im übrigen eine einseitige Berücksichtigung der europäischen Interessen eine wirkliche Sanierung der Hafenstadt nur erschweren würde. Die Einführung der Rassentrennung lehnte er aus Überzeugung ab. Er nannte sie «politisch falsch und unwissenschaftlich». ${ }^{14}$

Gerade die Rassentrennung war es jedoch, die den meisten anderen Zeitgenossen als schnellster und billigster Weg zur dringlichen Verbesserung der Gesundheitsverhältnisse für die Europäer in Afrika erscheinen wollte. Innert kürzester Zeit wurde sie zur bestimmenden Norm, die nach anfänglichen Diskussionen kaum je mehr kritisch hinterfragt wurde ${ }^{15}$. Wissenschaftliche Erkenntnisse, rassistische Vorurteile, politische Motive und 
wirtschaftliche Interessen gingen hierbei eine für die Zeit charakteristische Verbindung ein. Die wissenschaftliche Begründung der Forderung nach einer systematischen Trennung der Rassen findet sich erstmals in einem Bericht der beiden vom britischen Malaria-Komitee 1900 nach Westafrika und Indien ausgesandten Militärärzte J. W. W. Stephens und S. R. Christophers. Ähnlich wie vor ihnen der deutsche Bakteriologe Robert Koch stellten sie bei ihren Forschungen an Orten mit endemischer Malaria fest, daß vor allem ein Großteil der Kinder infiziert war und daß Schwere und Häufigkeit der Infektion mit zunehmendem Alter sanken ${ }^{16}$. Damit war der Nachweis erbracht, daß die Einheimischen sich im Laufe ihres Lebens eine gewisse Widerstandskraft gegen Malaria erwarben. Dies wiederum erklärte, weshalb erwachsene Einheimische weniger anfällig waren für Malaria als gleichaltrige Europäer und weshalb Europäer an Orten erkrankten, wo die erwachsene einheimische Bevölkerung gesund war. Die frühere, für die Denkmuster der Aufklärung kennzeichnende Ansicht, daß die Einheimischen gesund, die Malaria mithin wie andere Krankheiten von den Europäern selbst eingeschleppt werde, hatte sich als falsch erwiesen. Widerlegt schien auch die von Charles Darwin vorgebrachte Vermutung einer angeborenen Immunität der Neger gegen die Malaria, was er mit ihrer dunklen Hautfarbe in Zusammenhang gebracht hatte ${ }^{17}$. Wie sich später herausstellte, hatte Darwin nicht ganz unrecht, denn ein beträchtlicher Teil der Afrikaner ist Träger des Sichelzellgens und damit des anomalen Hämoglobins $\mathrm{S}$, das sie tatsächlich gegen die Auswirkungen einer Plasmodiumfalciparum-Infektion der tropischen Malaria widerstandsfähiger macht als Personen mit normalen Bluteigenschaften. Doch diese Erkenntnis datiert erst aus den 1950er Jahren.

Aus der Tatsache, daß die einheimische Bevölkerung und namentlich die Kinder wichtige Zwischenwirte für die Malaria-Plasmodien waren und gefährliche Infektionsherde darstellten, folgerten Stephens und Christophers - und die Fachwelt schloß sich ihnen an -, daß der Kampf gegen die Malaria auch den Schutz der Gesunden vor den menschlichen Zwischenwirten einschließen müsse, wenn er erfolgreich geführt werden sollte. Und, die eigene Argumentation verengend, schlossen sie, die Segregation sei der einzige Ausweg vor einer Infektion. Demgegenüber nannten sie die von MacGregor vorgebrachten Einwände gegen die Rassentrennung «falschsentimentale Ideen». ${ }^{18}$

Spätestens dieser Seitenhieb läßt aufhorchen. Er drängt die Frage auf, ob nicht ihre in wissenschaftlichem Gewande daherstolzierenden Ratschläge 
auf Entscheiden beruhen, die mehr durch den gesellschaftlichen Standort der Autoren als durch deren eigene wissenschaftliche Untersuchung begründet war. In der Tat wird in der erwähnten Verengung der Argumentation auf eine monokausale Erklärung ein Bruch sichtbar, der in diesem besonderen Fall mit dem damals virulenten Rassismus in Zusammenhang gebracht werden kann, darüber hinaus aber auf das grundsätzliche erkenntnistheoretische Problem der Standortgebundenheit wissenschaftlichen Erkennens hinweist. In der Geschichtswissenschaft hat eben dieses Problem zum Postulat geführt, daß der Historiker seinen gesellschaftlichen Standort reflektieren und für den Leser einsehbar machen müsse. Es ist erstaunlich, wie wenig derartige Überlegungen im Bereich der Medizin Eingang gefunden haben, obwohl doch auch die Vertreter dieser Wissenschaft mit ähnlichen Problemen konfrontiert sind, und zwar spätestens dann, wenn sie Handlungsanweisungen entwerfen. Es wäre natürlich unhistorisch, von den Tropenmedizinern der Jahrhundertwende ein derart geläutertes Verhältnis zum eigenen Tun zu verlangen, weil entsprechende Postulate erst später gestellt worden sind. Doch gerade die Geschichte der Malaria-Prophylaxe wirft ein besonders grelles Licht auf die gesellschaftliche Bindung medizinischer Forschung bzw. die Gefahren sozialpolitischer Blindheit der Forscher. Denn an der gesellschaftlichen Vermittlung des Segregationsrezeptes im Kampf gegen die Malaria kann kein Zweifel bestehen.

Sie wird bereits in der Anlage der entsprechenden Forschung deutlich. Während nämlich die hohe Sterblichkeit unter den Europäern verdiente Aufmerksamkeit fand, haben zum Beispiel Stephens und Christophers die Frage nach der Sterblichkeit der Einheimischen gar nicht erst gestellt. Und Hinweise auf Kindersterblichkeit als Folge von Malaria sucht man in ihren Berichten vergeblich. Im Gegenteil, sie vermitteln den Eindruck, als ob die Malaria-Infektion bei einheimischen Kindern ohne alle gesundheitlichen Schäden verlaufe: «Allgemein gesprochen sehen die Kinder mit sehr zahlreichen Parasiten im Blut nicht krank aus. Sie tollen umher oder besuchen die Schule und zeigen keine der wohlbekannten Symptome einer MalariaAttacke. ${ }^{19}$ Erst Jahre später, als der anhaltende Arbeitermangel in vielen Kolonien die Frage nach dem generativen Verhalten der Kolonisierten zu einem wirtschaftlich bedeutsamen Problem gemacht hatte, fiel der Blick der Ärzte auch auf die sehr hohe Kindersterblichkeit, worauf denn nicht länger verborgen blieb, daß sie zu einem guten Teil auf die Malaria zurückzuführen sei $^{20}$.

Im weiteren hat Robert Koch trotz ähnlicher Befunde wie Stephens und 
Christophers niemals den Ruf nach einer Rassentrennung erhoben, für die er, wie er sagte, wenig Symphathie hatte. Indessen glaubte er im Chinin ein Allheilmittel zu erkennen, wobei er betonte, das Chinin müsse auch den ärmeren Schichten, mithin auch den Afrikanern, zugänglich gemacht werden ${ }^{21}$. Sodann ist bezeichnend, daß in Europa selbst niemand je auf die Idee gekommen ist, im Kampf gegen die hiesige Malaria die systematische, staatlich verordnete Trennung zweier Bevölkerungsteile in Angriff zu nehmen. Und wenn schließlich der Segregationsvorschlag von Stephens und Christophers auf den ersten Blick in der Tradition des aus der Zeit der Miasma-Theorie stammenden Ratschlags zum Meiden ungesunder Orte zu stehen scheint, der in Indien sowie in Sierra Leone zur Anlage von Höhenstationen und einer De-facto-Segregation geführt hatte, so bestehen doch bedeutsame Unterschiede, die nicht nur den Paradigma-Wechsel in der Medizin, sondern darüber hinaus den Wandel der Werte im zeitgenössischen Denken aufdeckte. Denn fortan ging es nicht mehr um das Meiden bestimmter Naturräume, sondern um das Meiden bestimmter Menschengruppen. Schlimmer noch: Wenn man sich in der Zeit tropenmedizinischer Unschuld durch das Fernbleiben von möglichen Krankheitsherden zu schützen gesucht hatte, so bedeutete der wissenschaftlich fundierte Rat der Mediziner nun das Aussperren sowohl der wirklichen wie der möglichen Keimträger aus dem Verband der Gesunden, wobei die Dichotomie gesund-krank deckungsgleich war mit der in der Rassenlehre der Zeit begründeten vermeintlichen Dichotomie Europäer-Neger, die wiederum in der kolonialen Situation dem Gegensatzpaar Kolonisierende-Kolonisierte bzw. Herrscher und Beherrschte entsprach.

Die tiefe Menschenverachtung dieses Denkansatzes wird deutlich in den begleitenden Überlegungen zum Segregationsvorschlag, die der deutsche Tropenmediziner Friedrich Plehn in der von ihm verfaßten Tropenhygiene anstellt. In diesem weitverbreiteten Handbuch aus dem Jahre 1902 schreibt er, daß ein um 300-400 Schritt abgesetztes «Farbigenlager geradezu eine Art Sicherheitsventil für das Europäerlager darstellen [werde], insofern die Mücken und vor allem die Anophelesmücken die ihnen in kaum zu übertreffender Weise gebotene Bequemlichkeit und Behaglichkeit in den Arbeiterhütten nur ausnahmsweise mit den ihren Bedürfnissen weit weniger Rechnung tragenden Verhältnissen im Europäerlager vertauschen werden».22 Plehn betrachtete folglich die Einheimischen mit ähnlichen Augen wie jene Hirten ihr Vieh, von denen eingangs die Rede war. Und Plehns Gedanken sind keinesfalls die Verirrung eines einzelnen, denn in den Zentren kolonialer 
Durchdringung, in den Städten eben, ist die Segregation nach der Jahrhundertwende in eine generationenüberdauernde räumliche Ordnung übergeführt worden, die die obgenannten Gegensätze in die Zukunft festschrieb. Das war zumindest in den historisch gewachsenen Städten der Küste mit tiefgreifenden Umwälzungen verbunden.

Ein besonders instruktives Beispiel gibt die Geschichte der Hafenstadt Duala in Kamerun, das von 1884 bis zum Ersten Weltkrieg unter deutscher Kolonialherrschaft stand. In vorkolonialer Zeit hatten die am Ort tätigen Europäer, allesamt Händler, bis auf eine Ausnahme stets auf abgetakelten Schiffen im Wuri-Ästuar gewohnt und das Festland nur in geschäftlichen Angelegenheiten betreten. Die im Gegensatz zu den Missionaren, die seit 1845 eine Station in einem Duala-Dorf unterhielten. Im Gefolge der kolonialen Eroberung zogen auch die übrigen Europäer aufs Festland, und zwar hauptsächlich auf die klimatisch begünstigte, etwas erhöht gelegene Joß-Platte, wo sie von den Einheimischen Land für die Errichtung von öffentlichen Gebäuden, Warenlagern, Geschäftshäusern und Wohnbauten erwarben. Daraus ergab sich eine Stadt, in der 1913379 Weiße und rund 22000 Afrikaner neben- und miteinander wohnten und lebten. 1910 jedoch legten die Ortsbehörden ein Projekt vor, das die Umwandlung der JoßPlatte in eine Europäerstadt und die Enteignung bzw. die Aussiedlung der dort ansässigen Einheimischen in neue Quartiere an der Peripherie vorsah. Europäerstadt und Afrikaner-Quartiere sollten durch eine 1 Kilometer breite Freizone getrennt werden, entsprechend der Flugweite der Anopheles-Stechmücken. Jeder Europäer erhielt das Recht, drei schwarze Bedienstete bei sich unterzubringen. Alle anderen Afrikaner aber sollten die Europäerstadt nurmehr tagsüber betreten dürfen. Vorbild dafür war eine entsprechende Regelung im belgischen Kongo. Die Gesamtkosten dieses Projekts wurden auf nicht weniger als 5,6 Millionen Mark geschätzt ${ }^{23}$.

Die Gründe für diesen Entscheid sind vielfältig. Es spielten sowohl wirtschaftliche wie politische und strategische, aber auch kulturelle Motive eine wichtige Rolle. Genannt sei das Bestreben, das «Negerdorf» in eine moderne Stadt umzuwandeln, als Aushängeschild deutschen kolonialen Unterfangens, zudem als Großhafen und damit Angelpunkt wilhelminischer Weltpolitik ${ }^{24}$. Im Zentrum der offiziellen Begründungen standen freilich 


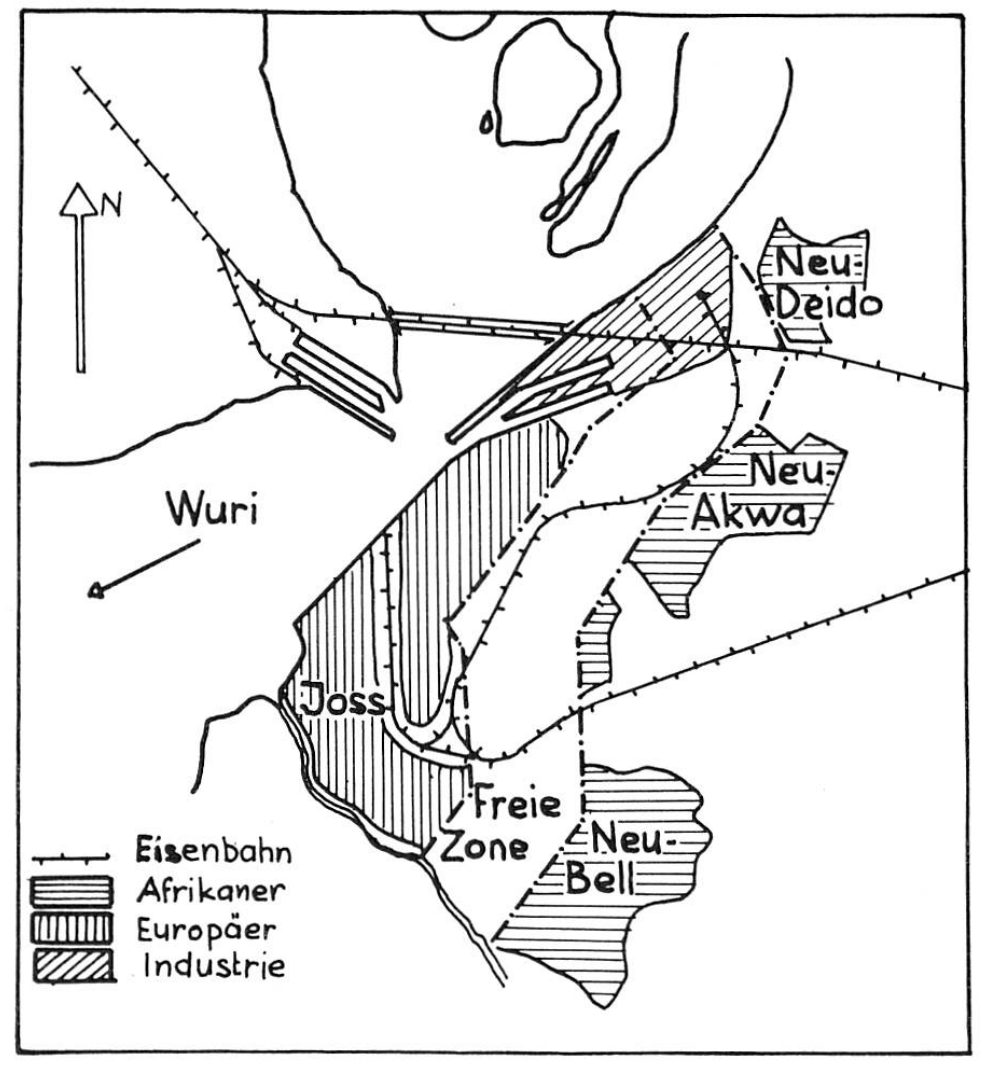

Stadtplanung Duala 1910

andere, nämlich tropenhygienische Argumente. Offensichtlich war das Segregationspostulat unter den Zeitgenossen bereits so weit verankert, daß die Behörden darin ein geeignetes Argument sehen konnten, um ihre Pläne vor der Öffentlichkeit über Partikularinteressen hinauszuheben und um die Zustimmung aller politischen Kräfte in der Heimat zu gewinnen. Die Verantwortlichen beriefen sich namentlich auf ein Gutachten des Regierungsarztes Professor Dr.med. Hans Ziemann, der sich zwar der sozialen Aspekte der Malaria-Frage sehr wohl bewußt war, der jedoch unter Hintanstellung eben dieser Argumente schon im Jahre 1900 an wissenschaftlichen Kongressen in Europa eine strikte Rassentrennung als sichersten Weg gepriesen hatte, um Duala für die «Europäer zu einer relativ ganz gesunden Tropenstadt zu machen, wo man ohne Chinin leben kann».$^{25}$ Die von Koch vorgeschlagene Chinin-Methode hielt er für «gänzlich undurchführbar», weil sie schädliche Nebenwirkungen habe und weil die Bevölkerung in den Städten viel zu stark fluktuiere, als daß eine allgemeine Verabreichung von Chinin möglich wäre. Die Kostenfrage klammerte er aus. Hingegen äußerte er sich dahingehend, daß die «Eingeborenen» weder willens noch fähig seien, den Nutzen eines systematischen Kampfes gegen die Malaria zu begreifen. 
Insbesondere meinte er, die Neger in Unterguinea seien eben weniger intelligent als jene in Oberguinea. Die Karriere von Horton und anderen Leuten aus Unterguinea kannte er offensichtlich nicht. Zudem verwies er auf entsprechende Mißerfolge seinerseits in Kamerun, allerdings ohne jeden genauen Beleg. In Wirklichkeit haben die Deutschen in Kamerun keinerlei großangelegte Kampagne gegen die Malaria begonnen; ein entsprechendes Sanitätsgesetz war lange in Diskussion, wurde jedoch nie erlassen. Wie vieles andere blieb der Kampf gegen die Malaria lange der Initiative einzelner überlassen. Ihnen fehlten in der Regel aber nicht nur die Mittel, sondern auch die nötige Geduld, was sie immer wieder zur Gewaltanwendung verleitete. Daß die konfliktgeladene politische Situation in Duala ihrerseits ein Hindernis für Neuerungen war, wollten sie nie verstehen. Im übrigen hielten sich nicht einmal alle Europäer an die von den Tropenärzten immer und immer wieder empfohlenen Schutzmaßnahmen ${ }^{26}$. Gerade dies aber war für die Deutschen ein zusätzlicher Grund, die Segregation voranzutreiben.

Doch so sehr sie sich um eine tropenmedizinische Begründung bemühten, - selbst die epidemiologischen Überlegungen der Ärzte waren von rassistischem Gedankengut durchtränkt. Zwei weitere Regierungsärzte führten in ihrem Gutachten folgende Beanstandungen auf: die unhygienischen Wohnverhältnisse vieler Einheimischer, die ständig schwelenden Feuer in ihren Häusern, der «ekelerregende Gestank» ihrer Speisen, ihr lautes Reden und ihr lautes Freizeitverhalten, ihr Eigengeruch, die «für deutsche Augen ungewohnte» Sitte der Frauen, ihre Haare im Freien pflegen zu lassen, und schließlich die vermeintliche Gefahr für die sittliche Erziehung der weißen Kinder, die sich aus dem engen Kontakt zu afrikanischen Altersgenossen ergebe: «Die weißen Kinder, so warnten sie, lernen gewöhnlich spielend die Negersprache, die ihnen besonders auf geschlechtlichem Gebiete Dinge mitteilt, vor denen unsere Kinder zu hüten unsere heilige Pflicht ist.» ${ }^{27}$ Die Autoren nannten das Rassenhygiene. So abstrus und willkürlich zusammengewürfelt die Argumente aus dem Nachhinein erscheinen, sie kreisen alle um den einen Punkt: den der Aussperrung der Afrikaner von der Welt der Kolonisierenden. Und so ist es nur folgerichtig, wenn dieselben Autoren darauf drängten, daß die Afrikaner in den neuen Quartieren nur Mattenhäuser im traditionellen Baustil errichten dürften. Häuser, die sich wie der Manga-Bell-Palast in ihrer Architektur an koloniale Vorbilder anlehnten und mithin Zeichen eines Wunsches nach der Übernahme europäischer Werte sind, wollten sie verboten wissen, da sie nur die, wie sie es nannten, «Afterkultur» verstärken und die Afrikaner immer mehr 
zu «Hosenniggern» werden ließen. Kurz: sie gaben die Rassentrennung auch als Schutz vor einer kulturellen Entfremdung der Afrikaner aus. In Wirklichkeit sind die Darlegungen der beiden Ärzte ein Zeugnis dafür, wie die Kolonialherren sich ihre Wilden konstruierten, um die Afrikaner leichter beherrschen zu können.

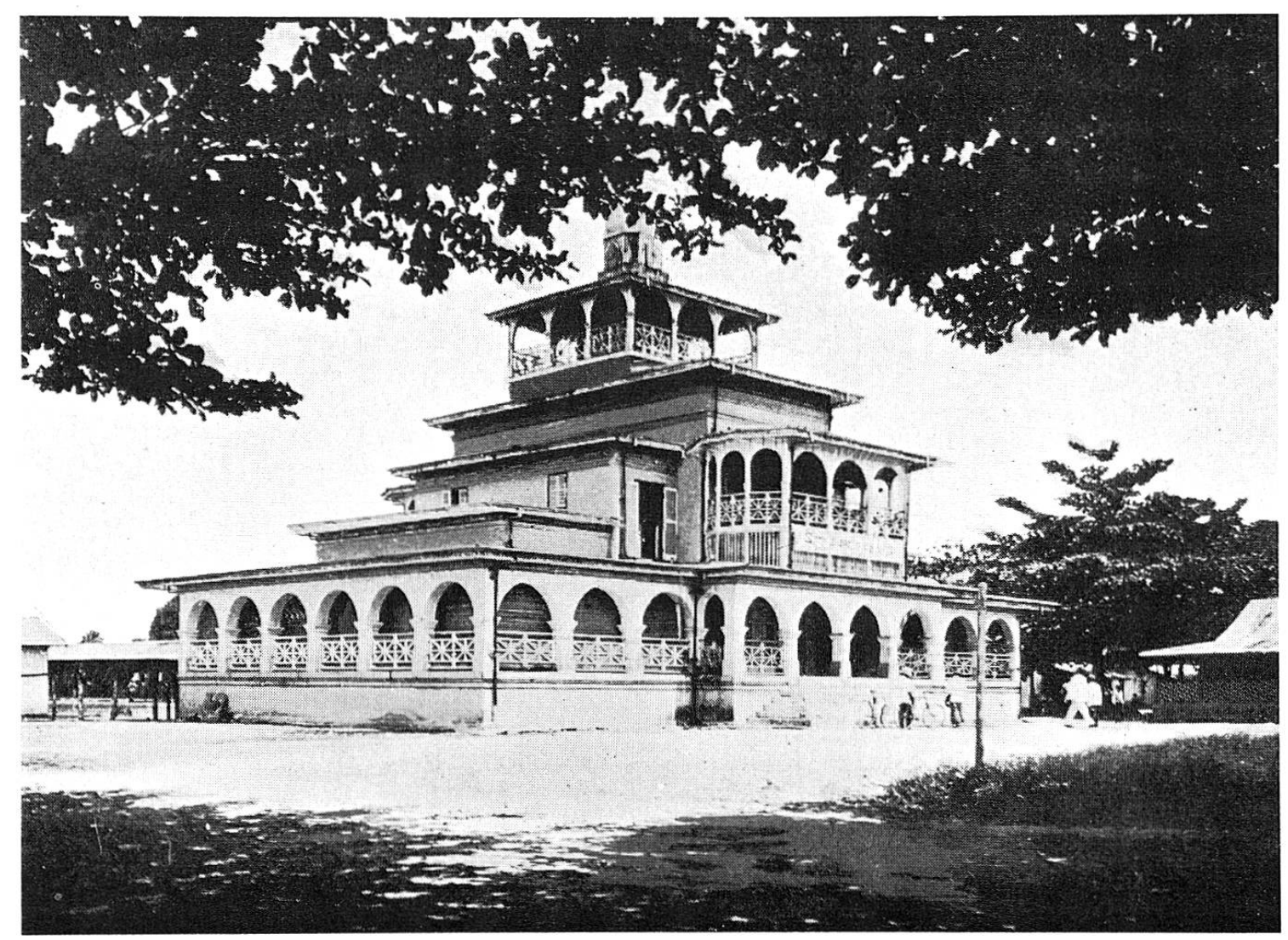

Haus des Duala Manga (sogenannter Manga-Bell-Palast)

Anderseits verweist ihre Argumentation auch auf die tiefsitzende Angst der Kolonisierenden vor der Fremdheit der Umgebung in den Tropen mit ihren Verlockungen und Gefahren. Oder in der brutalen Sprache der Zeit: vor der Verkafferung. Gemeint ist damit nichts anderes als die Anpassung des einzelnen an seine Umgebung, was gerade in der Fremde Voraussetzung für sein Überleben ist, zugleich aber die Grundlagen der Kolonialherrschaft untergraben mußte. Denn diese beruhte, wie jede nicht-demokratische politische Ordnung, auf möglichst großer sozialer Distanz zwischen Herrschern und Beherrschten. Paradigmatisch für die Tendenz zur Anpassung waren die Mischehen mit einheimischen Frauen, die die Deutschen denn 
auch unter Aufbringung aller möglichen und unmöglichen Gründe bekämpften. Es liegt auf der Hand, daß eine rassisch segregierte Stadt ein geeignetes Mittel war, um diesen Tendenzen entgegenzuwirken, indem sie die Fremden ausschloß und die Kolonisierenden zum sozialen Zusammenschluß zwang, sei es durch die Intensivierung der gegenseitigen Kontakte, durch die Mitnahme ihrer deutschen Frauen oder die Errichtung eines heimischen Vorbildern nachempfundenen Vereinslebens. In die gleiche Stoßrichtung gehört die Verankerung eines Sozial- und Wohlstandsgefälles, denn es galt der Grundsatz, daß erst der Besitz «den Menschen zum Menschen macht», während da, «wo aus der Hand in den Mund und nur für den nächsten Augenblick gelebt werden muß ... Kultur nicht möglich sei, dem Weißen so wenig wie dem Kaffer».. ${ }^{28}$

Insgesamt wird die Doppelbödigkeit des Vormundschafts- und Schutzarguments dann offensichtlich, wenn man sich vergegenwärtigt, daß 1. die Deutschen gerade die Mattenhäuser als Infektionsgefahr einschätzten, 2. die neuen Quartiere zum Teil in sumpfigem Überschwemmungsgebiet lagen, 3. mit der Umsiedlung begonnen wurde, noch bevor die nötige Infrastruktur (vor allem Wasserversorgung) errichtet war, und daß 4. die sogenannte Afterkultur der Duala auch deshalb so negativ eingeschätzt wurde, weil gerade sie die Duala instand stellte, mit den Deutschen von gleich zu gleich zu verkehren und ihre Rechte zu verteidigen. Die Duala wehrten sich nicht nur mit passivem Widerstand gegen die drohende Enteignung, sondern schrieben Eingaben um Eingaben an die Behörden in Duala und in Deutschland; zudem versicherten sie sich der Dienste eines deutschen Rechtsanwalts. Darüber aber kam es zu einem offenen politischen Konflikt, der nur durch den Ausbruch des Ersten Weltkrieges beendet wurde, freilich nicht, bevor die Deutschen 1914 den Oberhäuptling der Duala hingerichtet hatten.

Dieser politische Konflikt kann hier nicht mehr erörtert werden. Statt dessen sei nochmals auf die verhängnisvollen Zusammenhänge zwischen Malaria-Prophylaxe und Städtebau, zwischen Wissenschaft und Politik um die Jahrhundertwende hingewiesen. Die sozialpolitische Blindheit vieler Ärzte oder ihre Befangenheit in den rassistischen Vorurteilen der Zeit machten, daß die wissenschaftliche Revolution im Bereich der MalariaForschung sich in sozialem Rückschritt auswirkte, so daß auch Mediziner zu Vorkämpfern der Rassentrennung wurden. Im kolonialen Städtebau hat dieser Rassismus ein steinernes Denkmal erhalten, das bis heute die soziale Wirklichkeit in Afrika prägt. 


\section{Anmerkungen}

${ }^{1}$ L. J. Bruce-Chwatt, The challenge of malaria: Crossroads or impasse?, Wood 1978, S.27-48. Über den jeweils neuesten Stand der Malariabekämpfung unterrichtet regelmäßig die Chronique OMS.

${ }^{2}$ Einen Überblick über die Entwicklung der Sterberaten der Engländer im kolonialen Westafrika seit dem 19. Jahrhundert geben L. J. Bruce-Chwatt und Joan M. Bruce-Chwatt, Malaria and yellow fever, in: E.E.Sabben-Clare, D. J.Bradley, A.Kirkwood (Hrsg.), Health in tropical Africa during the colonial period, Oxford 1980, S. 143-162.

${ }^{3}$ Sorgfältig abwägend ist die Übersicht von August Hirsch, Die allgemeinen acuten Infectionskrankheiten vom historisch-geographischen Standpunkt und mit besonderer Berücksichtigung der Ätiologie, 2. Auflage, Stuttgart 1881 (Handbuch der historisch-geographischen Pathologie), Kap. 7. Neuere Darstellungen mit reichem historischem Material: Hans Ziemann in: Carl Mense (Hrsg.), Handbuch der Tropenkrankheiten, Bd.5, Leipzig 1917, und vor allem: Gordon Harrison, Mosquitoes, malaria and men: A history of the hostilities since 1880, London 1978.

${ }^{4}$ Vgl. Robert Koch, Ärztliche Beobachtungen in den Tropen (Vortrag vor der Deutschen Kolonialgesellschaft, Abt. Berlin-Charlottenburg, vom 9. Juni 1898), in Julius Schwalbe (Hrsg.), Gesammelte Werke von Robert Koch, Leipzig 1912, Bd.2, 1. Teil, S. 342. Im folgenden zitiert als Gesammelte Werke 2/1.

${ }^{5}$ C.P. Hull, The Europeans in India; or, Anglo-Indian's Vade-Mecum ..., to which is added a medical guide for Anglo-Indians, by R.S. Mair, 3. Auflage, London 1878, S.206f.

${ }^{6}$ James Africanus Horton, The diseases of tropical climates and their treatment, 2. Auflage, London 1879, S. 18.

7 R.S. Mair in: Hull, op.cit., S. 272 ff.

${ }^{8}$ Eine eingehendere Darstellung findet sich in Harrison, op. cit., Kap. 2-12. Verblüffend ist, wie sehr der Wettstreit der Forscher dem damaligen Wettlauf der europäischen Mächte um weltpolitischen Einfluß und Vorrang gleicht. Und natürlich neigte jede Nation dazu, den Beitrag ihrer Forscher zur Kenntnis der Malaria-Ätiologie als den entscheidenden darzustellen.

${ }^{9}$ Alle Zitate nach Jacob Lind, Versuch über die Krankheiten, denen Europäer in heißen Climaten unterworfen sind. Nebst der Methode, ihre gefährlichsten Folgen zu verhüten, Riga 1773, S.214f. James Lind ist vor allem bekannt durch seine revolutionären Erkenntnisse hinsichtlich der diätetischen Verursachung des Skorbuts: vgl. seinen Treatise on scurvy, London 1754.

${ }^{10}$ Zur Biographie: Christopher Fyfe, Africanus Horton 1835-1883. West African scientist and patriot. New York 1972.

${ }^{11}$ Seine Werke im einzelnen: The medical topography of the West Coast of Africa, London 1859; Geological constitution of Ahanta, Gold Coast, Freetown 1862; Political economy of British West Africa. The African's view of the negro's place in nature, London 1865; Physical an medical climate and meteorology of the West Coast of Africa, London 1867; West African countries and peoples, British and native. With the requirements necessary for establishing that self-government recommended by the committee of the House of Commons, 1865, and a vindication of the African race, London 1868; Guinea worm, or Dracunculus, London 1868; Lettres on the political condition of the Gold Coast, London 1870, und das bereits genannte Diseases of tropical climates. 
${ }^{12}$ Er führt diese Auseinandersetzung in den beiden Büchern Political economy und West African countries and peoples.

${ }^{13}$ Angelo Celli, The campaign against malaria in Italy, in: Journal of Tropical Medicine and Hygiene, Bd.11, Heft 7 (1908), S.107. Vgl. auch seine monographische Darstellung: Die Malaria nach den neuesten Forschungen, 2. Auflage, Berlin 1913.

${ }^{14}$ William MacGregor, A lecture on malaria, in: British Medical Journal [BMJ], Dec. 20 (1902), S. 1893. Ders., Notes on anti-malarial measures now being taken in Lagos, in: Ibid., Sept. 14 (1901), S. 680 ff. Ganz ähnlich die Argumentation des Principal Medical Officer von Lagos, Henry Strachan, in BMJ, Sept. 17 (1904), S.637 ff. Das Werk von MacGregor ist gewürdigt worden von Raymond E.Dumett, The campaign against malaria and the expansion of scientific medical and sanitary services in British West Africa, 1898-1910, in: African Historical Studies, Bd.1, Heft 2 (1968), S.153-197, und von R.B.Joyce, Sir William MacGregor, Melbourne 1971.

${ }^{15} \mathrm{Vgl}$. die äußerst rege Diskussion über Malaria-Prophylaxe im $B M J$ vor dem Ersten Weltkrieg. Bezeichnend ist auch, daß Albert Plehn, Beiträge zur Kenntnis von Verlauf und Behandlung der tropischen Malaria in Kamerun, Berlin 1896, noch keinerlei Segregationsvorschlag enthält, dies im Gegensatz zu den nach 1900 erschienenen Büchern zum Thema, wie etwa der maßgeblichen Synopsis von Ronald Ross, The prevention of malaria, London 1911. Ross und MacGregor standen sich in vielem sehr nahe, wie aus Ronald Ross, Memoirs with a full account of the great malaria problem and its solutions, London 1923, S. $445 \mathrm{ff}$., hervorgeht; hinsichtlich der Segregation vertraten sie dennoch gegensätzliche Standpunkte.

${ }^{16}$ J.W.W.Stephens, S.R.Christophers, The malarial infection of native children, in: Royal Society, Reports to the Malaria Committee, Third series, London 1900, S. 4-14.

${ }^{17}$ Charles Darwin, Die Abstammung des Menschen, Stuttgart 1966, S.253 ff. Englische Erstauflage: London 1871.

${ }^{18}$ Zit. nach J. W. W. Stephens' Rede am 72. Jahrestag der British Medical Association 1904, in: BMJ, Sept. 17 (1904), S.631. Vgl. aber auch J. W. W.Stephens, S. R. Christophers, The segregation of Europeans, in: Royal Society, op. cit., S.21-24, und dieselben, The native as the prime agent in the malarial infection of Europeans, in: Royal Society, Further reports to the Malaria Committee, London 1900, S. 3-19.

${ }^{19}$ J. W. W. Stephens, S. R. Christophers, The segregation of Europeans, op. cit., S.22. In ihrer gemeinsam verfaßten Monographie The practical study of malaria and other blood parasites, London 1903, S.266, bezeichnen sie die Malaria sogar als «normal condition of childhood».

${ }^{20}$ In diesem Sinne Jaeger, Beiträge zur Rassenhygiene aus dem Bezirk Ebolowa (Südkamerun), in: Archiv für Schiffs- und Tropenhygiene, 16/10 (1912), S.325-336, und L. Külz, Grundzüge der kolonialen Eingeborenenhygiene, Leipzig 1911 (Beihefte zum Archiv für Schiffs- und Tropenhygiene, Bd.15, Heft 8). Külz war ein besonders aufgeklärter Arzt im Kolonialdienst, der auch entsprechend erfolglos blieb in seinen Bemühungen um eine bessere ärztliche Versorgung der Afrikaner. Vor ihnen haben bereits W. MacGregor und R. Koch auf hohe Kindersterblichkeit im Zusammenhang mit endemischer Malaria hingewiesen, in: BMJ, Dec. 20 (1920), S.1890, bzw. im Zweiten Bericht über die Tätigkeit der Malaria-Expedition (1900), der wiederabgedruckt ist in: Gesammelte Werke 2/1, S. 399.

${ }^{21}$ Robert Koch, Address on malaria to the Congress at Eastbourne (1901), in: Gesammelte Werke 2/1, S.450. Vgl. außerdem seinen Dritten Bericht über die Tätigkeit der Malaria- 
Expedition (1900) und die Zusammenfassende Darstellung der Ergebnisse der MalariaExpedition (1900), beide in: Ibid., S.404-411, 420-434.

${ }^{22}$ Friedrich Plehn, Tropenhygiene mit spezieller Berücksichtigung der deutschen Kolonien, Jena 1902, S.216.

${ }^{23}$ Denkschrift über die Enteignung und Verlegung der Eingeborenen in Duala (Kamerun), in: Verhandlungen des Reichstags, XIII. Legislaturperiode, I. Session, Bd. 305, Anlagen zu den Stenographischen Berichten, Berlin 1914, Aktenstück Nr. 1576.

${ }^{24} \mathrm{Vgl}$. Ralph A.Austen, Duala versus Germans in Cameroon: economic dimension of a political conflict, in: Revue française d'histoire d'Outre-Mer, 64/237 (1977), S.477-497, und Adolf Rüger, Die Duala und die Kolonialmacht 1884-1914, in: Helmuth Stoecker (Hrsg.), Kamerun unter deutscher Kolonialherrschaft, Bd.2, Berlin (Ost) 1968, S.220-257.

${ }^{25}$ Gutachten des Regierungsarztes Professor Dr.Ziemann über die Notwendigkeit der Entfernung der Eingeborenen aus der Nähe der Europäer in Duala, in: Verhandlungen des Reichstags, op. cit., S.3306. Außerdem ders., Malaria prevention in uncultivated districts, in: BMJ, Oct. 14 (1907), S. 1046-1049.

${ }^{26}$ Das mag nicht zuletzt damit zusammenhängen, daß die Moskitotheorie keineswegs sofort allgemeine Anerkennung fand. Wie schwer sich selbst ein Teil der Ärzteschaft mit den Erkenntnissen von Ross und anderen abfand, zeigt der Artikel eines Dr. Braddock, Some notes on malarial fever as seen in the jungle, in: New York Medical Journal, Aug. 17 (1907). Demgegenüber wäre auf die Tatsache hinzuweisen, daß die Missionen ihre anfangs erschreckend hohen Sterblichkeitsraten vermittels konsequenter Prophylaxe, aber ohne Segregationsmaßnahmen, ganz wesentlich senken konnten. Vgl. R. Fisch, Die Wirkung der Malariaprophylaxe bei den Missionsangestellten in Kamerun, Berlin 1914 (Beihefte zum Archiv für Schiffs- und Tropenhygiene, Bd.18, Heft 4).

${ }^{27}$ Alle Zitate nach Ph. Kuhn, Noetel, Gutachten über die Notwendigkeit der Verlegung der Eingeborenen von Duala, in: Verhandlungen des Reichstags, op. cit., S. $3320 \mathrm{f}$.

${ }^{28}$ Verkafferung, in: Kamerun-Post (Duala), Nr.41 vom 24. Mai 1913. Zum Gesellschaftsleben der Europäer in der Kolonie: W.Langheld, Die Lebensbedingungen der Europäer in Kamerun, in: Die Woche, Nr.38 (1910), S. 1591-1593, und Mansfeld, Beim Gouverneur von Kamerun, in: Ibid., Nr.31 (1910), S. 1315-1318.

\section{Summary}

Malaria is one of the most widespread and most vicious endemic diseases of the tropics. Not surprisingly the history of malaria prophylaxis and colonial history are closely interwined. It was only at the turn of the century, in the haydays of colonial expansion in Africa, that the aetiology of malaria was discovered by men such as Laveran, Ross, Koch, Christophers, Stephens and others. Their truly revolutionary discoveries could have opened the way to rational and efficient programs of malaria prophylaxis and malaria eradication. But under the sociopolitical conditions of the day they but furthered racist policies in that they seemed to justify racial segregation in colonial towns. The city of Duala in Cameroon is a case where this policy was brought to its extreme, where the scientific paradigm was transposed into a spatial structure that was to stay as a monument to racism and to the dangers of sociopolitical blindness of the men of science.

PD Dr.phil. Albert Wirz, Historisches Seminar, Künstlergasse 16, 8006 Zürich 